\title{
Comparison of clinical methods for the phenotypic detection of Methicillin resistant Staphylococcus aureus: Disc diffusion methods with Brilliance ${ }^{\text {TM }}$ MRSA agar
}

\author{
Bashir Ahmad $^{1 \star}$, Johar Jamil ${ }^{1}$ and Jawad Ahmed ${ }^{2}$ \\ ${ }^{1}$ Centre of Biotechnology and Microbiology University of Peshawar, Pakistan. \\ ${ }^{2}$ Institute of Basic Medical Sciences, Khyber Medical University, Peshawar, Pakistan.
}

Accepted 15 March, 2013

\begin{abstract}
This study was based on the comparison of revised guidelines of Clinical and Laboratory Standard Institute (CLSI), British Society for Antimicrobial Chemotherapy (BSAC) and Brilliance ${ }^{\mathrm{TM}}$ MRSA agar (Oxoid) for the phenotypic identification of methicillin resistant Staphylococcus aureus (MRSA). A total of 133 clinical isolates of $S$. aureus were tested using new CLSI, BSAC guidelines and Brilliance ${ }^{\mathrm{TM}}$ MRSA Agar. All the strains were tested according to prescribed guidelines of CLSI (M100-S20, 2010) and BSAC (10.2, 2011). The $30 \mu \mathrm{g}$ and $10 \mu \mathrm{g}$ cefoxitin (FOX) performed best for the detection of MRSA and gave $100 \%$ sensitivity and specificity. While $1 \mu \mathrm{g}$ oxacillin (OX) showed less effective results showing sensitivity and specificity of $96.8 \%$ and $100 \%$ respectively. The cefoxitin disc diffusion method appears to be a good option for the identification of MRSA. Brilliance ${ }^{\mathrm{TM}}$ MRSA agar when used for the detection of MRSA, showed sensitivity and specificity of $98.4 \%$ and $100 \%$ respectively. In conclusion the efficiency of FOX is very high and FOX disc diffusion method is the most efficient method for the identification of MRSA phenotypically and could be a second choice for the detection of MRSA.
\end{abstract}

Key words: Staphylococcus aureus, CLSI, BSAC, Chromogenic media, MRSA.

\section{INTRODUCTION}

Methicillin resistant Staphylococcus aureus (MRSA) is responsible for serious infections worldwide. In both hospital and community settings, MRSA is an increasing threat to immunocompromised and to the normal healthy individuals. The accurate identification of MRSA is the most important step in effective management of the infected patient. This can also help to minimize risk of its transmission (Appelbaum, 2007; Berger-Bachi and Rohrer, 2002).

In MRSA PBP2 protein (PBP2' or PBP2a) is encoded by mecA gene which is present in MRSA strains. These proteins have low tendency for methicillin and all other $\beta$ lactam antibiotics. Factors, such as growth conditions including temperature and osmolarity of the medium, may change the phenotypic identification of methicillin resistance in $S$. aureus. The accuracy of identification methods may be affected by these factors (Chambers, 1997).

Various methods are used for the phenotypic detection of MRSA including disc diffusion method. In disc diffusion 
method, CLSI (2007) recommends the utility of cefoxitin instead of oxacillin for the detection of MRSA (CLSI,2007). Cefoxitin disc is superior to oxacillin for two reasons, firstly the interpretation of zone is easy and secondly, it is more effective for the detection of mecAmediated resistance (Felten et al., 2002; Mimica et al., 2007; Pottumarthy et al., 2005; Swenson and Tenover, 2007; Velasco et al., 2005; Witte et al., 2007). It is difficult to prove the phenotypic detection of MRSA with disc diffusion method using special media, minimum inhibitory concentration (MIC) determination or agar break point methods. The heterogeneous methicillin resistance expression in many $S$. aureus strains has been described (Chambers, 1997). The number and heterogeneity level of these strains are increasing. The identification of such strains is a challenge even if special phenotypic methods are used (Brown, 2001). Therefore, for the determination of methicillin resistance in Staphylococci, mecA or PBP2 detection is considered to be a gold standard (Brown, 2001; Dufour et al., 2002 ; Gosbell et al., 2001; Swenson et al., 2001). However, in Pakistan most of the laboratories do not have the economic and technical capabilities for applying all these tests on the $S$. aureus isolates recovered in microbiology laboratories. Therefore, oxacillin disc diffusion method is used for the routine screening of MRSA.

Chromogenic media have been used for the last few years for the quick detection of microorganisms from clinical specimens (Felten et al., 2002). These media contain "chromogenic" substrate which is integrated into a solid-agar-based matrix. This substrate detects special enzyme(s) produced by the microorganism(s) which are the identification markers for microbes (Perry and Freydiere, 2007). Therefore, in contrast to other conventional methods, chromogenic media identify the pathogen by direct colony colour from the first culture. This is a time saving method which minimizes further sub-culturing for further biochemical testing until a result is obtained (Kumar et al., 2010).

This study aims to compare the sensitivity and specificity of oxacillin and cefoxitin disc diffusion methods by the revised CLSI (2010), BSAC (2011) guidelines and Brilliance $^{\mathrm{TM}}$ MRSA (Oxoid) agar for phenotypic identification of MRSA.

\section{MATERIALS AND METHODS}

Three different methods were compared for the identification of MRSA and MSSA. The S. aureus Oxford Staph ATCC 9144 was used as a negative control and MRSA BIG 0047 (collected at Harrogate District hospital, Harrogate UK, 2007) was used as a positive control. In total, 133 clinical isolates of $S$. aureus were tested.

All the $S$. aureus isolates were collected from two tertiary care hospitals of Peshawar, Khyber Teaching Hospital and Lady Reading Hospital. These isolates were tested by using OX $1 \mu \mathrm{g}$ disc on Muller Hinton Agar (MHA) (Oxoid, Basingstoke UK) without additional sodium chloride $(\mathrm{NaCl})$. A suspension of each $S$. aureus iso- late was prepared to a $0.5 \mathrm{McF}$ arland standard. All the strains were inoculated on MHA and were incubated for $24 \mathrm{~h}$ at $30^{\circ} \mathrm{C}$ in air. According to CLSI (M100-S20) guidelines, if the zone diameter was $\leq 10 \mathrm{~mm}$, the test strain was considered as MRSA. For FOX $30 \mu \mathrm{g}$ discs were used on MHA and incubated for $16-18 \mathrm{~h}$ at $33-35^{\circ} \mathrm{C}$. If the zone diameter was $\leq 21 \mathrm{~mm}$, then the test strain was considered as MRSA.

By using BSAC $(10.2,2011)$ method, all the 133 clinical isolates of $S$. aureus were tested by using FOX $10 \mu \mathrm{g}$ on Iso-Sensitest Agar (ISA) (Oxoid, Basingstoke UK) and OX $1 \mu \mathrm{g}$ on MHA (Oxoid, UK) supplemented with $2 \% \mathrm{NaCl}$. All the strains were inoculated on ISA for FOX and on MHA for OX and incubated at 35 and $30^{\circ} \mathrm{C}$ each for $18 \mathrm{~h}$, respectively. According to BSAC recommendations if the zone diameter was $\leq 21 \mathrm{~mm}$ for FOX and $\leq 14 \mathrm{~mm}$ for OX, then the test isolate was considered as MRSA.

Brilliance ${ }^{T M}$ MRSA Agar (Oxoid, Basingstoke UK) has been used for the phenotypic identification of MRSA. It is an opaque medium which contains new chromogen. Many staphylococci including $S$. aureus have phosphatase activity which gives blue color on this chromogenic medium. The growth of MSSA is inhibited by the cocktail of antibiotics present in this medium. It also contains many compounds that inhibit the phosphatase activity in other Staphylococci, thus increasing the level of sensitivity and specificity. Brilliance $^{T M}$ MRSA agar is supplied as pre-poured culture plates. It is used for the identification of MRSA in human specimens.

All the 133 clinical isolates of $S$. aureus were tested on this medium. Before inoculation, the medium was allowed to warm up to room temperature and then dried up in incubator at $37^{\circ} \mathrm{C}$. The agar screening plates were inoculated directly by picking isolated colonies of test isolate and incubated for $18-24 \mathrm{~h}$ at $37^{\circ} \mathrm{C}$. The denim blue color colonies were reported positive for MRSA.

\section{RESULTS}

A total of 133 clinical isolates of $S$. aureus were screened by all three methods. All isolates were recovered from the wounds of both in and out door patients of two tertiary hospitals of Peshawar, Pakistan.

By using CLSI guidelines, among 133 isolates, OX 1 $\mu \mathrm{g}, 61(45 \%)$ were MRSA and 70(52.6\%) were MSSA. Two isolates showed false negative results and gave intermediate zone diameters of $11 \mathrm{~mm}$. The specificity and sensitivity of OX $1 \mu \mathrm{g}$ was 100 and $96.8 \%$, respectively. In contrast FOX $30 \mu \mathrm{g}$ showed $100 \%$ sensitivity and $100 \%$ specificity respectively (Table 1 ). All the isolates showed the zone diameter less than $21 \mathrm{~mm}$.

Using BSAC recommendations, $63(47.36 \%)$ of the Staphylococci, were MRSA and 70(52.6\%) were MSSA. According to this guideline, FOX $10 \mu \mathrm{gg}$ gave $100 \%$ specificity and $100 \%$ sensitivity. By using the OX $1 \mu \mathrm{g}$ method, two isolates gave false negative results, showing zone diameter greater than $14 \mathrm{~mm}$ thus these two isolates were sensitive. Therefore, the OX $1 \mu \mathrm{g}$ showed $96.8 \%$ sensitivity and $100 \%$ specificity (Table 1 ).

Brilliance $^{\text {TM }}$ MRSA agar (Oxoid) was used for the phenotypic identification of MRSA (Figure 1) as described by Kumar et al. (2010). Among 133 isolates, 61(45\%) were MRSA and $72(54 \%)$ were MSSA. It gave false negative results for one isolate which did not show any growth, while it showed positive results for MRSA for both 
Table 1. Comparision of sensitivies and specificities of three methods used in this study.

\begin{tabular}{|c|c|c|}
\hline Method & Sensitivity (\%) & Specificity (\%) \\
\hline CLSI (FOX 30 $\mu \mathrm{g})$ & 100 & 100 \\
\hline$(\mathrm{OX} 1 \mu \mathrm{g})$ & 96.8 & 100 \\
\hline BSAC (FOX 10 $\mu \mathrm{g})$ & 100 & 100 \\
\hline$(O X 1 \mu \mathrm{g})$ & 96.8 & 100 \\
\hline Brilliance ${ }^{\mathrm{TM}}$ MRSA Agar & 98.4 & 100 \\
\hline
\end{tabular}

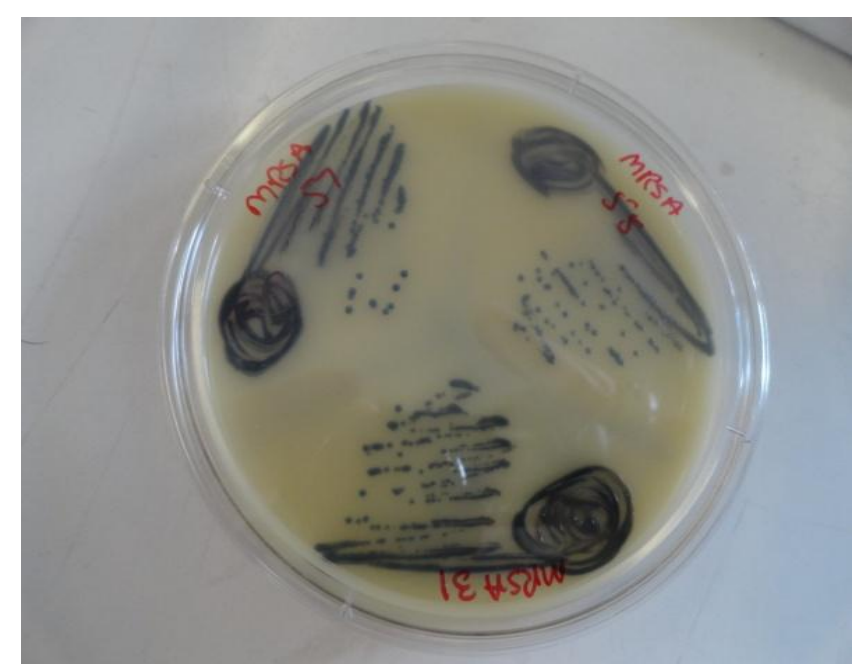

Figure 1. Morphological appearance of MRSA on BrillianceTM MRSA agar.

FOX (10 and $30 \mu \mathrm{g})$ discs. The sensitivity and specificity of Brilliance ${ }^{\mathrm{TM}}$ MRSA agar were $98 \%$ and $100 \%$ respectively (Table 1 ).

\section{DISCUSSION}

It is important to identify MRSA accurately because it will help in proper management of $S$. aureus born diseases. Many methods for the identification of MRSA have been developed but most of them are costly, time consuming and not useful enough for the correct detection of MRSA. (Kumar et al., 2010; Tiwari et al 2009).

The most reliable method for detecting MRSA isolates is the detection of mecA, however in routine clinical practice; most of the laboratories do not use molecular techniques for the detection of MRSA. Therefore, those phenotypic techniques are very important, which are capable to identify MRSA strains quickly and accurately. This will also ensure the appropriate antimicrobial therapy at appropriate time and prevent the spreading of such nasty MRSA strains in both health care and community settings. S. aureus with a mecA mediated resistance can Be detected by cefoxitin (Swenson et al., 2007). In 2009, new breakpoints for the FOX $30 \mu \mathrm{g}$ disc test for identification of mecA in $S$. aureus have been validated by CLSI (Nicole et al., 2009). Their results showed that FOX disc diffusion test is the best technique and a better alternate for the OX disc diffusion test due to its easy interpretation and high accuracy. Our study findings are similar to their findings. In the present study, FOX $10 \mu \mathrm{g}$ and $30 \mu \mathrm{g}$ showed that they are the best for phenotypic detection of MRSA. We have also found false negative results for detection of MRSA by using OX $1 \mu \mathrm{g}$ according to both CLSI (M100-S20) and BSAC guidelines. Skov et al. (2003) also found that for identification of MRSA, FOX disc diffusion test was usually good, with sensitivity and specificity of $100 \%$ and $99 \%$, respectively; which is similar to this study. They used FOX $30 \mu \mathrm{g}$ on ISA (Skov et al., 2003). In another study, Skov et al. (2005) found that using semi-confluent growth on ISA and MHA along with standard incubations conditions, performance of FOX 5 and $10 \mu \mathrm{g}$ discs was excellent for the identification of MRSA (Skov et al., 2005). In the present study the sensitivity and specificity were both $100 \%$ for FOX $10 \mu \mathrm{g}$, which is similar to the findings of Skov et al. (2005).

Several chromogenic media and other deferential MRSA selective agars have been used to identify MRSA within 18 to $24 \mathrm{~h}$ (Flayhart et al., 2005; Nahimana et al., 2006; Stoakes et al., 2006; Diederen et al., 2005; Nguyen Van et al., 2006). In this study we used Brilliance ${ }^{\mathrm{TM}}$ MRSA agar (Oxoid) for the detection of MRSA. The sensitivity and specificity were $98.4 \%$ and $100 \%$, respectively. In a study by Kumar et al. (2010) the sensitivity for Brilliance ${ }^{\mathrm{TM}}$ MRSA (Oxoid) was $97 \%$, which is similar to this study, and the specificity was $86 \%$ which was different from this study. This change in the specificity may be due to the difference in the collection of samples, clones circulating in different parts of the world and prevalence of MRSA (Van Loo et al., 2007). Another study modelled the effect of direct identification of MRSA carriage using a real-time PCR or chromogenic media on capture of patient isolation days (PIDs). The analysis showed that real time PCR captured $22.7 \%$ more PIDs and these more undesired PIDs were because of slightly low specificity as compared to chromogenic medium (Robicsek et al., 2008 ). Similar performance of a chromogenic medium and RT-PCR has also been reported in 
another study (Bischof et al., 2009). However, yet another study has reported that molecular techniques give quick results with high sensitivity (Paule et al., 2009).

Brilliance $^{\mathrm{TM}}$ MRSA agar (Oxoid) is also a good, cost effective medium for the detection of MRSA because cost of screening on conventional culture medium is similar to the cost of chromogenic medium. When downstream processing time and cost of the technician is considered, variations range from $1.4-1.7$ days, excluding time required for confirmatory test(s) (Nahimana et al., 2006). On the basis of advantages of chromogenic media, it should be encouraged in the laboratories for rapid screening of MRSA (Kumar et al., 2010). Similarly, in a study conducted by Verkade et al. (2009) Brilliance ${ }^{\mathrm{TM}}$ MRSA (Oxoid) has been found to be the best for the identification of MRSA. They used 788 isolates of staphylococci and after $20 \mathrm{~h}$ of incubation, the sensitivity and specificity were $99.6 \%$ and $97.3 \%$ respectively. For the screening of MRSA in less time, this new medium is highly sensitive. Our results are similar to those reported by Vekade et al. (2009). By using Brilliance ${ }^{\mathrm{TM}}$ MRSA (Oxoid), results can be obtained within one day. Further studies should be performed to find out the usefulness of Brilliance $^{\mathrm{TM}}$ MRSA (Oxoid) for the screening of MRSA directly from clinical specimens (Verkade et al., 2009).

In conclusion, FOX $10 \mu \mathrm{g}$ and $30 \mu \mathrm{g}$ were the best for the phenotypic detection of MRSA because their sensitivity and specificity were better than oxacillin. In most of the Pakistani labs oxacillin disc are still used for the detection of MRSA. It is therefore recommended that oxacillin discs should be replaced by the FOX discs test method because of its effectiveness. Brilliance ${ }^{\mathrm{TM}}$ MRSA agar is also effective and could be used as a second option for the direct inoculation of specimens in the laboratories.

\section{ACKNOWLEDGEMENT}

This work was funded by Higher Education Commission of Pakistan.

\section{REFERENCES}

Appelbaum P (2007). Microbiology of antibiotic resistance in Staphylococcus aureus. Clin. Infect. Dis. 45:165-170.

Berger-Bachi B and Rohrer S ( 2002). Factors influencing methicillin resistance in staphylococci. Arch. Microbiol. 178:165-171.

Bischof LJ, Lapsley L , Fontecchio K, Jacosalem D, Young C, Hankerd $R$, and. Newton DW (2009). Comparison of chromogenic media to BD Gene Ohm methicillin-resistant Staphylococcus aureus (MRSA) PCR for detection of MRSA in nasal swabs. J. Clin. Microbiol. 47:2281-2283.

Brown DF (2001). Detection of methicillin/oxacillin resistance in staphylococci. J. Antimicrob. Chemother. 48:65-70.

BSAC (2011). British society for Antimicrobial Susceptibility Testing. Version 10.2

Chambers HF (1997). Methicillin resistance in staphylococci: molecular and biochemical basis and clinical implications. Clin. Microbiol. Rev. 10:781-791.
CLSI (2010). Performance standards for antimicrobial susceptibility testing; 20th informational supplement M100-S20. CLSI, Wayne, PA.

CLSI (2007). Performance standards for antimicrobial susceptibility testing; 17th informational supplement. CLSI M100-S17. CLSI, Wayne, PA.

Diederen $B$, van Duijn I, van Belkum A, Willemse $P$, van Keulen $P$, Kluytmans J (2005). Performance of CHROMagar MRSA medium for detection of methicillin-resistant Staphylococcus aureus. J Clin Microbiol. 43:1925-1927.

Dufour P, Gillet Y, Bes M, Lina G, Vandenesch F, Floret D, Etienne J, Richet H (2002). Community-acquired methicillin-resistant Staphylococcus aureus infections in France: emergence of a single clone that produces Panton-Valentine leukocidin. Clin. Infect. Dis. 35:819-24.

Felten A, Grandry B, Lagrange PH, Casin I (2002). Evaluation of three techniques for detection of low-level methicillin-resistant Staphylococcus aureus (MRSA): a disc diffusion method with cefoxitin and moxalactam, the Vitek 2 system, and the MRSA-screen latex agglutination test. J. Clin. Microbiol. 40: 2766-2771.

Flayhart D, Hindler JF, Bruckner DA, Hall G, Shrestha RK Vogel SA, Richter SS, Howard W, Walther R, Carroll KC (2005). Multicenter evaluation of BBL CHROMagar MRSA medium for direct detection of methicillin-resistant Staphylococcus aureus from surveillance cultures of the anterior nares. J. Clin. Microbiol. 43:5536-5540.

Gosbell IB, Mercer JL, Neville SA, Chant KG, Munro R (2001). Community acquired non-multiresistant oxacillin-resistant Staphylococcus aureus (NORSA) in South Western Sydney. Pathol. 33:206-10.

Kumar SM, Abrahantes J, Lamme SC (2010). Evaluation of chromogenic media for detection of MRSA. J. Clin Microbiol . 48:1040-1046.

Mimica MJ, Berezin EN, Carvalho RL, Mimica IM, Mimica LM, Safadi MA, Schneider E, Caiaffa-Filho HH (2007). Detection of methicillin resistance in Staphylococcus aureus isolated from paediatric patients: Is the cefoxitin disk diffusion test accurate enough? Braz. J. Infect . Dis. 11:415-417.

Nahimana I, Francioli P, Blanc DS (2006). Evaluation of three chromogenic media (MRSA-ID, MRSA-Select and CHROMagar MRSA) and ORSAB for surveillance cultures of methicillin-resistant Staphylococcus aureus. Clin. Microbiol. Infect.12:1168-1174.

Nguyen Van JC, Kitzis MD, Ly A, Chalfine A, Carlet J, Ben Ali A. Goldstein F (2006). Detection of nasal colonization methicillinresistant Staphylococcus aureus: a prospective study comparing realtime genetic amplification assay vs. selective chromogenic media. Pathol Biol. 54:285-292.

Nicole MB, Van TT, Monson TA, Marshall SA, Warshauer DM (2009). Comparison of Cefoxitin and Oxacillin disc diffusion Methods for Detection of mecA-Mediated Resistance in Staphylococcus aureus in a large-scale study. J. Clin. Microbiol. 47: 217-219.

Paule SM, Mehta M, Hacek DM, Gonzalzles TM, Robicsek A, Peterson LR (2009). Chromogenic media vs real-time PCR for nasal surveillance of methicillin-resistant Staphylococcus aureus: impact on detection of MRSA-positive persons. Am. J. Clin. Pathol. 131:532539.

Perry JD and Freydiere AM (2007). The application of chromogenic media in clinical microbiology. J. Appl. Microbiol. 103:2046-2055.

Pottumarthy S, Fritsche TR, Jones RN (2005). Evaluation of alternative disk diffusion methods for detecting mecA-mediated oxacillin resistance in an international collection of staphylococci: validation report from the SENTRY antimicrobial surveillance program. Diag Microbiol Infect Dis. 51:57-62.

Robicsek A, Paule SM, Hacek DM, Kaul KL, Peterson LR (2008). Impact of test characteristics on an MRSA surveillance program, abstr. K-1704. Infectious Disease Society of America 46th Annual Meeting.

Skov R, Smyth R, Clausen M, Larsen AR, Moller NF, Liliequist BO, Kahlmeter G (2003). Evaluation of a cefoxitin $30 \mu \mathrm{g}$ disc on IsoSensitest agar for detection of methicillin-resistant Staphylococcus aureus. J. Antimicrob. Chemother. 52:204-207.

Skov R, Smyth R., Larsen AR, Moller NF, Kahlmeter G (2005). Evaluation of cefoxitin 5 and $10 \mu \mathrm{g}$ discs for the detection of methicillin 
resistance in staphylococci. J. Antimicrob. Chemother . 55:157-161.

Stoakes L, Reyes R, Daniel J, Lennox G, John MA, Lannigan R, and Hussain Z (2006). Prospective comparison of a new chromogenic media, MRSASelect, to CHROMagar MRSA and mannitol-salt medium supplemented with oxacillin or cefoxitin for detection of methicillin-resistant Staphylococcus aureus. J. Clin. Microbiol. 44:637-639.

Swenson JM, Williams PP, Killgore G, Hara CM, Tenover FC(2001). Performance of eight methods, including two new rapid methods, for detection of oxacillin resistance in a challenge set of Staphylococcus aureus organisms. J. Clin. Microbiol. 39:3785-8. 20.

Swenson, JM, Lonsway D, McAllister S, Thompson L, Jevitt L, Zhu W, Patel JB. (2007). Detection of mecA-mediated resistance using reference and commercial testing methods in a collection of Staphylococcus aureus expressing borderline oxacillin MICs. Diagn Microbiol Infect Dis.58:33-39.

Tiwari HK, Sapkota D, Das AK and Sen MR (2009). Assessment of different tests to detect Methicilin resistant Staphylococcus aureus. South East Asian J. Trop. Med. Public Health. 40:801-806.

Van Loo IH, van Dijk S, Verbakel-Schelle IAGM, Buiting AGM (2007). Evaluation of a chromogenic agar (MRSASelect) for the detection of meticillin-resistant Staphylococcus aureus with clinical samples in The Netherlands. J. Med. Microbiol. 56(4):491-494.
Velasco D, Tomas MM, Cartelle M, BecceiroA, Perez A, Molina F, Moure R, Villanueva R, Bou G (2005). Evaluation of different methods for detecting methicillin (oxacillin) resistance in Staphylococcus aureus. J Antimicrob Chemother. 55:379-382.

Verkade E, Elberts S, Verhulst C, Kluytmans J(2009). Performance of Oxoid Brilliance ${ }^{\mathrm{TM}}$ MRSA medium for detection of methicillin-resistant Staphylococcus aureus: an in vitro study. Eur J Clin Microbiol Infect Dis. 28:1443-1446.

Witte W, Pasemann B, Cuny C (2007). Detection of low-level oxacillin resistance in mecA-positive Staphylococcus aureus. Eur. J. Clin. Microbiol. Infect Dis. 13:408-412. 